\title{
On the Propagation of a Normal Shock Wave through a Layer of Incompressible Porous Material
}

\author{
R. Torrens and L.C. Wrobel* \\ Department of Mechanical Engineering, Brunel University, Uxbridge, UB8 3PH, UK
}

\begin{abstract}
A novel numerical formulation of the two-phase macroscopic balance equations governing the flow field in incompressible porous media is presented. The numerical model makes use of the Weighted Average Flux (WAF) method and Total Variation Diminishing (TVD) flux limiting techniques, and results in a second-order accurate scheme. A shock tube study was carried out to examine the interaction of a normal shock wave with a thin layer of porous, incompressible cellular ceramic foam. Particular attention was paid to the transmitted and reflected flow fields. The numerical model was used to simulate the experimental test cases, and their results compared with a view to validating the numerical model. A phenomenological model is proposed to explain the behaviour of the transmitted flow field.
\end{abstract}

Key words: Shock waves, shock tube, incompressible porous media, WAF method, TVD flux limiter

*Corresponding author: luiz.wrobel@brunel.ac.uk 


\section{Introduction}

Many researchers have contributed to the body of literature relating to wave propagation in porous media. Some of the earliest work by Biot (1956) present a mixture theory approach to the modelling of weak acoustic wave propagation in porous media, which has been used by various researchers to model both linear and non-linear problems. Baer (1988) and Powers et al (1989) present multiphase mixture theory models aimed at simulating the propagation of a compaction wave in a column of air-saturated granular porous media. In both cases, good agreement was obtained between the results of the numerical tests and experimental data for steady wave characteristics. Corapicoglu (1991) presents an extensive literature survey on work done in the field of mixture theory wave propagation modelling. This approach yields excellent results when modelling the propagation of linear waves in porous media. However, as the work of Rogg et al (1981) and Powers et al (1989) have shown, applying this technique to the propagation of nonlinear wave phenomena (i.e. shock waves) produces poor results.

Levy et al (1996) state that the processes involved in shock wave propagation in a porous medium should be modelled using the multiphase approach. This involves the definition of a system of governing equations describing the mass, momentum and energy processes in each phase, and the interactions between individual phases. Various researchers have adopted the macroscopic continuum approach to modelling flow in porous media, as opposed to the theory of mixtures. This involves the introduction of a macroscopic Representative Elementary Volume (REV) over which the extensive variables are averaged. The advantage of this method is that it yields information about the geometry of the porous medium that is not available using the mixture method. 
Hassanizadeh and Gray (1979a, 1979b, 1980) present a continuum approach technique to macroscopically average the mass, momentum and energy equations for an $n$-phase porous medium. The macroscopic balance equations for an abrupt pressure wave propagating through a porous domain were developed by Bear and Sorek (1990). The resulting two-phase system of macroscopic balance equations was evolved in time to reveal four distinct periods of behaviour. Firstly, a period of uniform pressure, temperature and stress distribution. This occurs at the instant the porous medium is subjected to the abrupt change in pressure. Secondly, a period of non-linear wave propagation that is dominated by convection. This is characterised by a sharp, welldefined compaction wave propagating in the porous medium. Thirdly, a period in which dissipative effects start to become apparent. The compaction wave in the porous layer starts to be more affected by the interaction with the internal surfaces of the porous medium. Finally, a period dominated by dissipative terms. The viscous dissipation caused by friction on the internal surfaces characterises the flow. This work was extended by Levy et al (1995) to provide a more accurate representation of the momentum and energy processes resulting from the action of the fluid on the internal surfaces of the porous medium. This was achieved by including the Forchheimer term in the macroscopic balance equations, which had been neglected in the model presented by Bear and Bachmat (1990).

Several numerical and experimental studies have been performed to examine the effects of shock wave reflection from a sample of porous medium mounted at the end of the driven section of a shock tube. Levy et al (1993) present a shock tube study to examine a shock wave impinging on a sample of rigid ceramic foam. From the experimental 
observations, Levy et al (1993) deduced a phenomenological model to account for the behaviour of the shock wave reflected from the front edge of the porous medium sample. This model suggested that, as the shock wave impinges on the front edge of the porous medium, a proportion of the wave would be reflected while the rest would propagate inside. The compaction wave penetrating the porous medium could be viewed as a sharp front that sweeps through it. As this compaction wave propagates, parts of it would be reflected from the internal surfaces of the porous medium. Some of these reflected wavelets would exit from the front edge of the porous medium and serve to strengthen the initially reflected wave. Levy et al (1996) presented a theoretical model of the shock tube problem studied in Levy et al (1993). A numerical formulation of this model was derived by the application of the high-order finite difference method of Harten (1983). Numerical solutions were obtained based on the experimental test cases given in Levy et al (1993).

Torrens and Wrobel (2002) presented a numerical model, based on the application of the Weighted Average Flux (WAF) method of Toro (1992), to the theoretical model of shock wave flow in porous medium discussed in Levy et al (1996). The numerical model was used to generate solutions for a shock tube problem where the domain was occupied entirely by a porous medium. In order to increase the accuracy of the solutions while preserving stability, various TVD flux limiting functions were applied to the WAF based formulation. It was found that the Superbee limiter of Roe (1983) was the most suited to the application.

Two new studies are presented in this paper. The first is an experimental study of a layer of porous media mounted in the middle of the driven section of a shock tube. The second 
is a numerical study using the model presented by Torrens and Wrobel (2002) to simulate the experimental test cases. A comparison between the numerical and experimental results is presented, and a phenomenological model is proposed to account for the transmitted wave behaviour as the shock wave leaves the porous medium.

\section{Governing Equations}

In this section, the one-dimensional system of multiphase equations governing the mass, momentum and energy processes in the porous medium are presented. The complexity of the porous medium geometry renders standard microscopic level continuum analysis impractical. Applying volume and areal averaging techniques to the equations governing the microscopic transport of extensive quantities (mass, momentum and energy) over a macroscopic REV results in six macroscopic balance equations describing the fluid and solid mass, momentum and energy processes.

The full system of macroscopic balance equations, obtained under a set of simplifying assumptions discussed by Levy et al (1996) and Torrens and Wrobel (2002), along with the constitutive relationships for stress and strain in the solid matrix and the equation of state for the gas, are given in the form:

Mass

$$
\begin{gathered}
\frac{\partial \phi \rho_{f}}{\partial t}+\frac{\partial \phi \rho_{f} u_{f}}{\partial x}=0 \\
\frac{\partial(1-\phi) \rho_{s}}{\partial t}+\frac{\partial(1-\phi) \rho_{s} u_{s}}{\partial x}=0
\end{gathered}
$$


where $u_{\alpha}$ and $\rho_{\alpha}$ are the velocity and density of the $\alpha$ phase ( $\alpha$ may be $f$ for the fluid phase or $s$ for the solid phase), and $\phi$ denotes the macroscopic porosity of the porous medium, given by:

$$
\phi=1-\frac{\rho_{\text {bulk }}}{\rho_{S}}
$$

where $\rho_{\text {bulk }}$ is the bulk density of the porous medium.

Momentum

$$
\begin{aligned}
& \frac{\partial \phi \rho_{f} u_{f}}{\partial t}+\frac{\partial \phi \rho_{f} u_{f}^{2}}{\partial x}=-C_{T} \frac{\partial \phi P}{\partial x}+C_{T} P \frac{\partial \phi}{\partial x}-C_{F} \phi \rho_{f}\left|u_{f}-u_{s}\right|\left(u_{f}-u_{s}\right) \\
& \frac{\partial(1-\phi) \rho_{s} u_{s}}{\partial t}+\frac{\partial(1-\phi) \rho_{s} u_{s}^{2}}{\partial x}=-C_{T} \frac{\partial(1-\phi) P}{\partial x}+\frac{\partial \sigma_{s}^{\prime}}{\partial x}-C_{T} P \frac{\partial \phi}{\partial x}+C_{F} \phi \rho_{f}\left|u_{f}-u_{s}\right|\left(u_{f}-u_{s}\right)
\end{aligned}
$$

where $P$ is the pressure, $C_{T}$ is the tortuosity constant describing the directional cosines of the fluid path through the porous medium, and $C_{F}$ is the Forchheimer constant.

Energy

$$
\begin{array}{r}
\frac{\partial \phi \rho_{f}\left(c_{f} T_{f}+\frac{1}{2} u_{f}^{2}\right)}{\partial t}+\frac{\partial \phi \rho_{f} u_{f}\left(c_{f} T_{f}+\frac{1}{2} u_{f}^{2}\right)}{\partial x}=-C_{T} \frac{\partial \phi u_{f} P}{\partial x}+C_{T} P \frac{\partial \phi}{\partial x} \\
-C_{F} \phi \rho_{f}\left|u_{f}-u_{s}\right|\left(u_{f}-u_{s}\right) u_{s} \\
\begin{array}{r}
\frac{\partial(1-\phi) \rho_{s}\left(c_{s} T_{s}+\frac{1}{2} u_{s}^{2}\right)}{\partial t}+\frac{\partial(1-\phi) \rho_{s} u_{s}\left(c_{s} T_{s}+\frac{1}{2} u_{s}^{2}\right)}{\partial x}=-C_{T} \frac{\partial(1-\phi) u_{s} P}{\partial x}+\frac{\partial u_{s} \sigma_{s}^{\prime}}{\partial x} \\
-C_{T} P \frac{\partial \phi}{\partial x}+C_{F} \phi \rho_{f}\left|u_{f}-u_{s}\right|\left(u_{f}-u_{s}\right) u_{s}
\end{array}
\end{array}
$$

where $T_{\alpha}$ and $c_{\alpha}$ are the temperature and specific heat capacity at constant volume in the $\alpha$ phase. 
The effective stress in the solid matrix may be expressed in the following form:

$$
\sigma_{s}^{\prime}=L_{1} \varepsilon-L_{2} c_{s}\left(T_{s}-T_{s 0}\right)
$$

where $L_{1}$ and $L_{2}$ are the Lamé constants for the solid matrix, $T_{s 0}$ is its initial temperature and $\varepsilon$, the macroscopic strain as a function of porosity, is defined by:

$$
\varepsilon=1-\frac{r_{s}}{r_{s 0}}
$$

where $r_{s}$ and $r_{s 0}$ are the mass fraction of the solid and its initial value, respectively. Finally, the second constitutive law - the equation of state for the fluid - is written in the form

$$
P=\rho_{f} R T_{f}
$$

where $R$ is the gas constant for air.

The previous system of governing equations may be represented in vector form as follows:

$$
\mathbf{U}_{t}+[\mathbf{F}(\mathbf{U})]_{x}=\mathbf{Q}
$$

where the conserved variable vector, $\mathbf{U}$, is given by:

$$
\mathbf{U}=\left[\begin{array}{llllll}
r_{f} & r_{s} & m_{f} & m_{s} & E_{f} & E_{s}
\end{array}\right\rfloor
$$

in which $r_{\alpha}$ is the mass fraction in the $\alpha$ phase, $m_{\alpha}$ is the momentum in the $\alpha$ phase and $E_{\alpha}$ is the energy in the $\alpha$ phase. These conserved variables are defined for the fluid phase by:

$$
r_{f}=\phi \rho_{f}, m_{f}=r_{f} u_{f} \text { and } E_{f}=r_{f}\left(c_{f} T_{f}+\frac{1}{2} u_{f}^{2}\right)
$$


and for the solid phase by:

$$
r_{s}=(1-\phi) \rho_{s}, m_{s}=r_{s} u_{s} \text { and } E_{s}=r_{s}\left(c_{s} T_{s}+\frac{1}{2} u_{s}^{2}\right)
$$

The flux vector $\mathbf{F}(\mathbf{U})$ is given by:

$$
\mathbf{F}(\mathbf{U})=\left[\begin{array}{c}
m_{f} \\
m_{s} \\
\frac{m_{f}^{2}}{r_{f}}+C_{T} \phi P \\
\frac{m_{s}^{2}}{r_{s}}-\sigma_{s}^{\prime}+\left(1-C_{T} \phi\right) P \\
\frac{m_{f}}{r_{f}}\left(E_{f}+C_{T} \phi P\right) \\
\frac{m_{s}}{r_{s}}\left(E_{s}-\sigma_{s}^{\prime}+\left(1-C_{T} \phi\right) P\right)
\end{array}\right]
$$

where the effective stress in the solid is given by:

$$
\sigma_{s}^{\prime}=L_{1}\left(\frac{r_{s 0}-r_{s}}{r_{s 0}}\right)-L_{2}\left(\frac{E_{s}}{r_{s}}-\frac{E_{s 0}}{r_{s 0}}-\frac{m_{s}^{2}}{2 r_{s}^{2}}+\frac{m_{s 0}^{2}}{2 r_{s 0}^{2}}\right)
$$

and the fluid pressure is given by:

$$
\phi P=(\gamma-1)\left[E_{f}-\frac{m_{f}^{2}}{2 r_{f}}\right]
$$

where $\gamma$ is the ratio of specific heats for air, and the subscript $s 0$ denotes an initial value. Note that equations (15) and (16) are the constitutive laws governing the stress-strain relationships in the solid and fluid phases, respectively. 
The source vector, $\mathbf{Q}$, is given by:

$$
\mathbf{Q}=\left[\begin{array}{c}
0 \\
0 \\
C_{T} P \frac{\partial \phi}{\partial x}-C_{F} r_{f}\left|\frac{m_{f}}{r_{f}}-\frac{m_{s}}{r_{s}}\right|\left(\frac{m_{f}}{r_{f}}-\frac{m_{s}}{r_{s}}\right) \\
-C_{T} P \frac{\partial \phi}{\partial x}+C_{F} r_{f}\left|\frac{m_{f}}{r_{f}}-\frac{m_{s}}{r_{s}}\right|\left(\frac{m_{f}}{r_{f}}-\frac{m_{s}}{r_{s}}\right) \\
\frac{m_{s}}{r_{s}}\left(C_{T} P \frac{\partial \phi}{\partial x}-C_{F} r_{f}\left|\frac{m_{f}}{r_{f}}-\frac{m_{s}}{r_{s}}\right|\left(\frac{m_{f}}{r_{f}}-\frac{m_{s}}{r_{s}}\right)\right) \\
\left.\frac{m_{s}\left(-C_{T} P \frac{\partial \phi}{\partial x}+C_{F} r_{f}\right.}{r_{s}} \mid \frac{m_{f}}{r_{f}}-\frac{m_{s}}{r_{s}}\left(\frac{m_{f}}{r_{f}}-\frac{m_{s}}{r_{s}}\right)\right)
\end{array}\right]
$$

Levy et al (1996) state that the porosity gradient term in the source vector is assumed to be small. If it were to be large, the nature of the system of equations represented by equation (10) would not be hyperbolic. This would preclude the formation of nonlinearities in the solution domain.

\section{Solution Strategy}

The complexity of the macroscopic balance equations described in the previous section renders a solution by analytical means impossible. This means that a solution may only be achieved by the application of a numerical method. This section presents the numerical techniques that were applied to solve equation (10).

The one-dimensional domain was divided into $n$ computational cells and the shock tube problem initial data, denoted by $\mathbf{U}^{n}$ (where $n=0$ as the shock tube diaphragm is removed), was applied piecewise constant to each cell. In order to solve equation (10), the 
following explicit conservative finite difference formula was used to update the initial data at time level $n$ to the new values at time level $n+1$ :

$$
\mathbf{U}_{i}^{n+1}=\mathbf{U}_{i}^{n}-\lambda\left(\mathbf{F}_{i+\frac{1}{2}}^{n}-\mathbf{F}_{i-\frac{1}{2}}^{n}\right)+\Delta t \mathbf{Q}_{i}^{n}
$$

where $\mathbf{U}_{i}^{n+1}$ and $\mathbf{U}_{i}^{n}$ denote the conserved variable vectors at time $n$ and time $n+1$ in the $i$ th computational cell, $\mathbf{F}_{i \pm \frac{1}{2}}^{n}$ denote the intercell flux components upstream and downstream of the $i$ th computational node, and $\mathbf{Q}_{i}^{n}$ denotes the source vector on the $i$ th computational node, at time $n$.

The intercell flux and source vector terms must be calculated before the solution may be updated. In respect of the latter, it is simply a matter of inserting the values from the conserved variable vector, $\mathbf{U}_{i}^{n}$, into the source vector (17). However, in order to evaluate the flux terms, the Riemann problem must be solved at each intercell boundary. This was achieved by the application of the WAF method of Toro (1992), coupled with the Superbee flux limiter method of Roe (1983), to impose a TVD condition on the solution. 


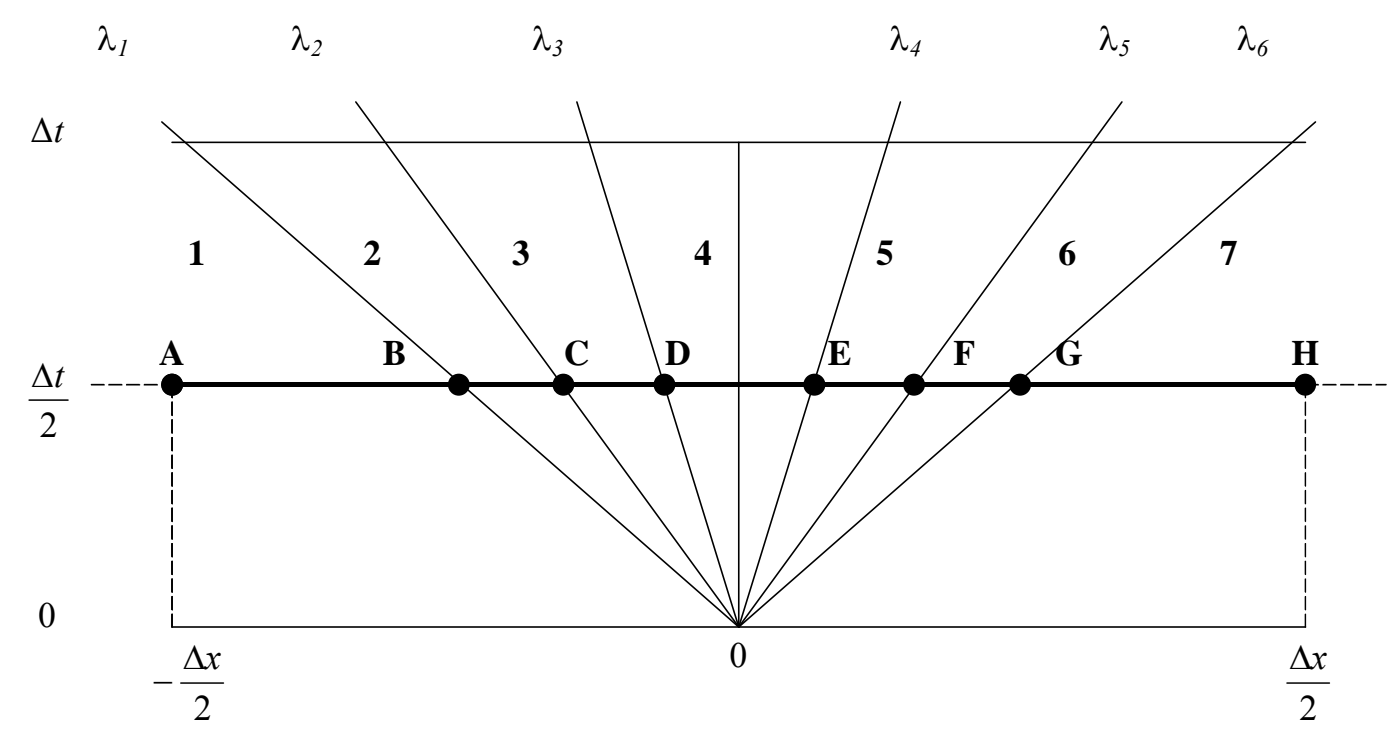

Figure 1: Characteristic field for (1) on $x$-t diagram

Consider the $x$-t plot shown in figure 1 , which illustrates the characteristic field for the Riemann problem between cells $i$ and $i+1$. There are six characteristics corresponding to the shock, contact and rarefaction waves propagating in the fluid and solid phases. Regions 1 and 7 represent the known states in the $i$ and $i+1$ computational cells. Regions 2 to 6 are the star states where the conserved variables are unknown. In order to implement the WAF and Superbee methods, an explicit knowledge of the conserved variables and fluxes in the star regions is required. In order to achieve this, the method of Roe (1981) was adopted. This involves linearising equation (10) by the introduction of a parameter vector averaged across the known states $\mathbf{U}_{1}$ and $\mathbf{U}_{7}$. This results in a constant Jacobian matrix, $\tilde{\mathbf{A}}$. The eigenvalues, eigenvectors and wave strengths of $\tilde{\mathbf{A}}$ were found symbolically using Mathematica, and are presented in full by Torrens and Wrobel (2002). Each of the six characteristics in the solution domain illustrated by figure 1 has an 
eigenvalue, an eigenvector and a wave strength associated with it. The star state conserved variables were calculated using the following expression:

$$
\mathbf{U}_{p}=\mathbf{U}_{1}+\left.\sum_{j=1}^{p-1} \widetilde{\alpha}_{j} \widetilde{K}^{(j)}\right|_{p=2, \ldots, 6}
$$

where $\mathbf{U}_{l}$ denotes the known conserved variable vector in region $1, \widetilde{\alpha}_{j}$ denotes the Roe averaged wave strength for the $j$ th characteristic and $\widetilde{\mathbf{K}}^{(j)}$ denotes the Roe averaged eigenvector associated with the $j$ th characteristic. The star state fluxes were given by the following expression:

$$
\mathbf{F}_{p}=\mathbf{F}_{1}+\left.\sum_{j=1}^{p-1} \widetilde{\alpha}_{j} \widetilde{\lambda}_{j} \widetilde{K}^{(j)}\right|_{p=2, \ldots, 6}
$$

where $\mathbf{F}_{l}$ denotes the known flux vectors in region 1 and $\tilde{\lambda}_{j}$ denotes the Roe averaged eigenvalue of the $j$ th characteristic wave. The weighted average intercell flux is given by the sum of the flux vector in regions 1 to 7 multiplied by their respective weight. Figure 1 shows a line, $\mathbf{A H}$, subdivided into seven sections. The length of each of these sections corresponds to the weight of the section it is in. The following expression gives the WAF intercell flux:

$$
\mathbf{F}_{i+\frac{1}{2}}^{W A F}=\sum_{k=1}^{7} \beta_{k} \mathbf{F}_{k}
$$

where $\mathbf{F}_{k}$ denotes the flux in the $k$ th region and $\beta_{k}$ is the weight, which may be given by: 


$$
\beta_{k}=\frac{1}{2}\left(c_{k}-c_{k-1}\right)
$$

where $c_{k}$ is the Courant number of the $k$ th wave given by:

$$
c_{k}=\frac{\Delta t \tilde{\lambda}_{k}}{\Delta x}
$$

The second-order WAF intercell flux is given by:

$$
\mathbf{F}_{i+\frac{1}{2}}^{W A F}=\frac{1}{2}\left(\mathbf{F}_{1}+\mathbf{F}_{7}\right)-\frac{1}{2} \sum_{k=1}^{6} c_{k} \Delta \mathbf{F}^{(k)}
$$

Note that the flux jump term denoted by $\Delta \mathbf{F}^{(k)}$ may be replaced by the Roe averaged flux.

In order to prevent the appearance of oscillations in the solution, the Superbee flux limiter of Roe (1983) was introduced. This resulted in the following modification to the WAF flux:

$$
\mathbf{F}^{W A F}=\frac{1}{2}\left(\mathbf{F}_{1}+\mathbf{F}_{7}\right)-\frac{1}{2} \sum_{k=1}^{6} \operatorname{sgn}\left(c_{k}\right) \varphi_{k} \widetilde{\alpha}_{k} \tilde{\lambda}_{k} \widetilde{\mathbf{K}}^{(k)}
$$

where $\operatorname{sgn}\left(c_{k}\right)$ is the sign of the Courant number of the $k$ th characteristic, and $\varphi_{k}$ is the Superbee flux limiter function. The flux limiter is a function of two data-dependent parameters. These are the Courant number and the ratio of upwind to local change of the conserved variables across each wave. The latter is given by:

$$
r_{k}=\frac{\rho_{f}^{k+1}-\left.\rho_{f}^{k}\right|_{i-\frac{1}{2}}}{\rho_{f}^{k+1}-\left.\rho_{f}^{k}\right|_{i+\frac{1}{2}}}
$$

The Superbee flux limiter function may be described as follows: 


$$
\varphi(r)=\left\{\begin{array}{ccc}
1 & \text { if } & r \leq 0 \\
1-2(1-|c|) r & \text { if } & 0 \leq r \leq \frac{1}{2} \\
|c| & \text { if } & \frac{1}{2} \leq r \leq 1 \\
1-(1-|c|) r & \text { if } & 1 \leq r \leq 2 \\
2|c|-1 & \text { if } & r \geq 2
\end{array}\right.
$$

Validation of the WAF-based numerical model was carried out by performing numerical simulations of three test case scenarios, as illustrated in figure 2(a)-(c). Figure 2(a) shows a plain shock tube, Figure 2(b) shows a shock tube entirely occupied by porous media while Figure 2(c) shows a shock tube with a sample of porous medium mounted at the end of the shock tube driven section. The validation of the first two configurations was presented by Torrens and Wrobel (2002). The validation of the third configuration was carried out by comparing experimental results presented by Levy et al (1993) with solutions generated by the numerical model presented herein. The initial value problem for this configuration is shown below:

$$
\mathbf{U}\left(x, t_{0}\right)= \begin{cases}\mathbf{U}_{L} & x \text { lies to the left of the diaphragm } \\ \mathbf{U}_{R 1} & \text { where } x \text { lies to the right of the diaphragm in an one phase cell } \\ \mathbf{U}_{R 2} & x \text { lies to the right of the diaphragm in a two phase cell }\end{cases}
$$

In defining the initial value problem, it was assumed that the air in the shock tube is initially quiescent, the temperature in the solid and fluid phases is $293 \mathrm{~K}$ at all points in the domain, and the specific heat capacities of the solid and fluid are $718 \mathrm{~kJ} / \mathrm{kg} / \mathrm{K}$ and $870 \mathrm{~kJ} / \mathrm{kg} / \mathrm{K}$, respectively. The initial pressures and porous medium geometry coefficients are given in table 1 . This information was used to calculate the conserved variable vectors $\mathbf{U}_{L}, \mathbf{U}_{R l}$ and $\mathbf{U}_{R 2}$, which may be defined by: 


$$
\mathbf{U}_{L}=\left[\begin{array}{c}
4.17 \\
0 \\
0 \\
0 \\
877259.58 \\
0
\end{array}\right], \mathbf{U}_{R 1}=\left[\begin{array}{c}
1.2 \\
0 \\
0 \\
0 \\
253489.02 \\
0
\end{array}\right] \text { and } \mathbf{U}_{R 1}=\left[\begin{array}{c}
0.88 \\
548.1 \\
0 \\
0 \\
185129.12 \\
1.397 \times 10^{8}
\end{array}\right]
$$

The grid sensitivity of the present numerical scheme was examined in Torrens and Wrobel (2002). To effectively model a 1.6 metre long domain, occupied entirely by porous medium, at least 400 computational nodes were required. The total number of computational cells used in the solution to the system (10) with initial conditions (28) was 400 , with 50 of the cells being placed on the porous medium.

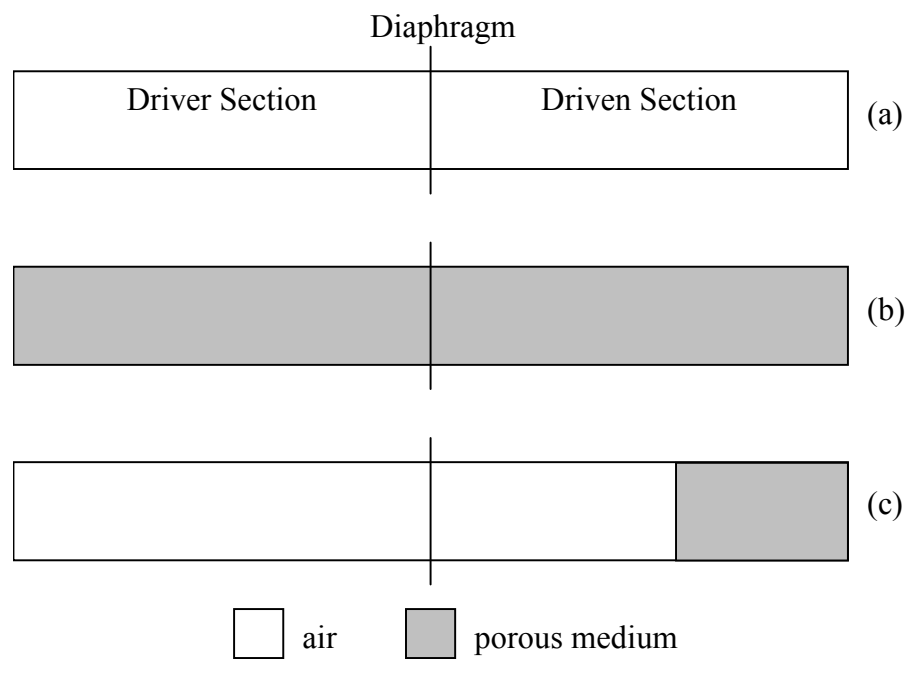

Figure 2: Validation configurations 


\begin{tabular}{|l|l|}
\hline Initial pressure in driver section & $351325 \mathrm{~Pa}$ \\
\hline Initial pressure in driven section & $101325 \mathrm{~Pa}$ \\
\hline Initial porosity & 0.73 \\
\hline Forchheimer constant & $300 \mathrm{~m}^{-1}$ \\
\hline Tortuosity constant & 0.7 \\
\hline
\end{tabular}

Table 1: Initial parameters for validation of configuration (c)

Figures 3 and 4 show pressure-time curves of the numerical solution to the problem defined by (29). These correspond to the two observation points at which the pressure was measured at each time step. These were positioned $10 \mathrm{~cm}$ behind and $10 \mathrm{~cm}$ ahead of the air/porous medium interface. The pressure history in figure 3 is initially at atmospheric pressure, as the observation point is positioned in the driven section. When the shock wave reaches it, the pressure jumps to approximately $180 \mathrm{kPa}$ (the magnitude of the initial shock wave). The same pressure is observed until the reflected shock wave from the air/porous medium interface reaches it. The pressure then jumps to approximately $250 \mathrm{kPa}$. These pressure values are consistent with the values predicted by Levy et al (1996). The shape and magnitude of the pressure-time curve in figure 4 also correspond well with those of Levy et al (1996). 


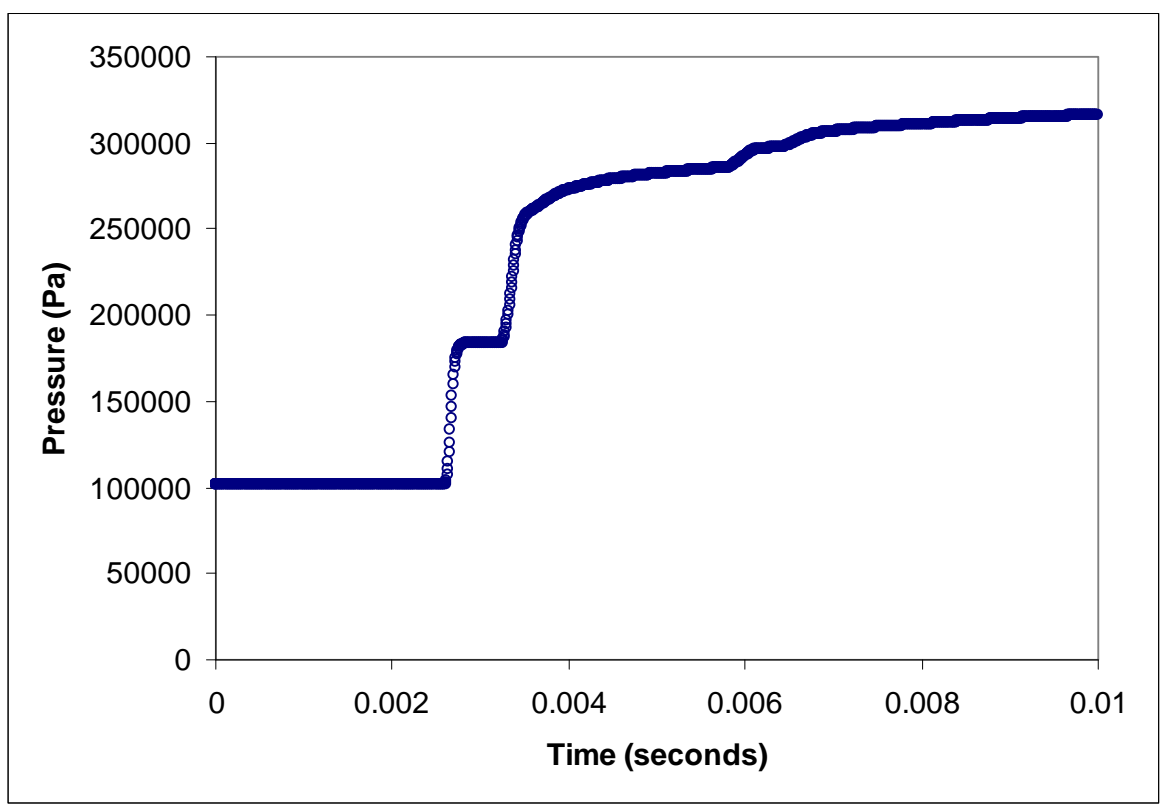

Figure 3: Pressure history for configuration (c) taken at an observation point just behind the air/porous medium interface

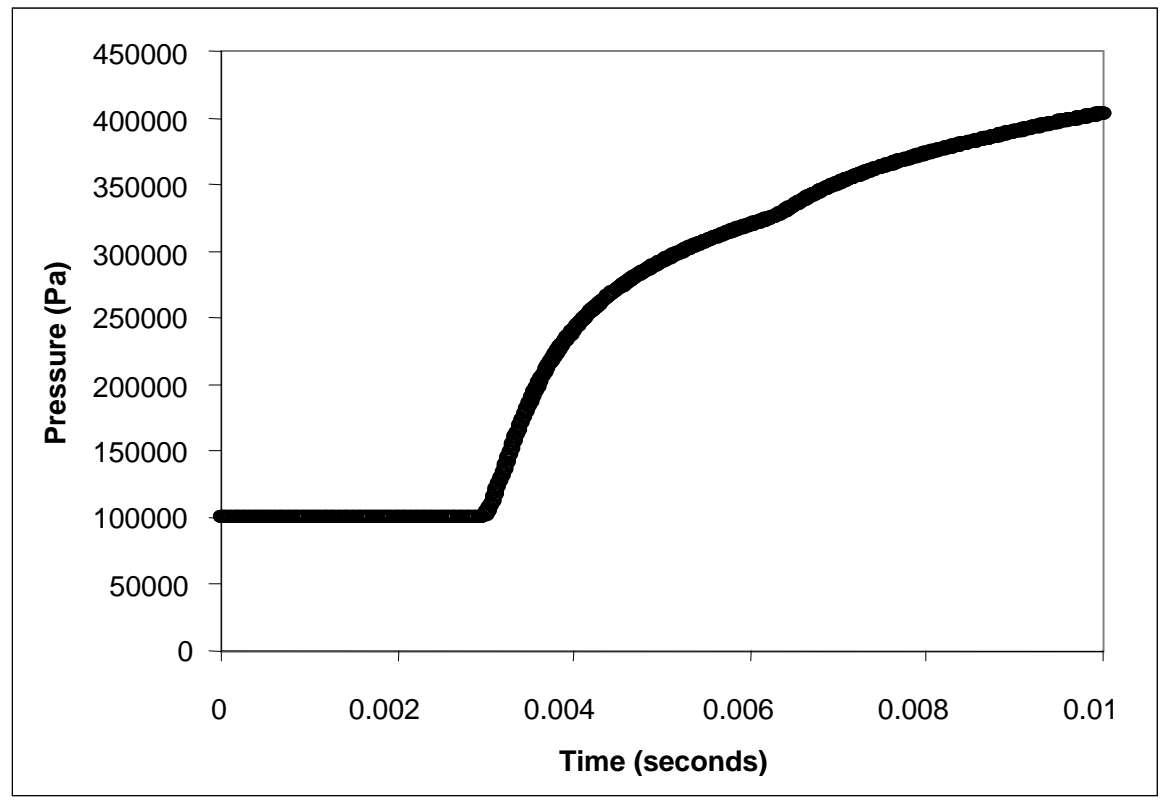

Figure 4: Pressure history for configuration (c) taken at an observation point just ahead of the air/porous medium interface 


\section{Experimental Study}

This section details the methods used to obtain experimental pressure histories of the shock wave reflected from the front edge of the porous medium layer and the wave transmitted from the back edge. The experimental study of shock propagation through a layer of porous medium was carried out in a shock tube with internal diameter of $55 \mathrm{~mm}$. Figure 5 shows a schematic of the shock tube, illustrating the position of the porous layer and the position of the pressure transducers used to obtain the pressure histories ahead of, and behind, the porous layer.

The two transducers used to measure the transient pressure were piezoelectric transducers manufactured by Kistler Instruments Ltd. Transducer 1 in figure 5, located 90mm behind the front edge of the porous layer, was a model 701a sensor while transducer 2, situated $90 \mathrm{~mm}$ ahead of the back face of the porous layer, was a model 7001 sensor. The use of these sensors was recommended by Kistler Instruments (2000). Both sensors have the same pressure range ( 0 to 250 bar) and sensitivity ( $80 \mathrm{pC} / \mathrm{bar})$.

The porous medium used in this experimental study was a rigid porous ceramic foam called Sivex, supplied by Pyrotek (UK) Limited. Three grades of the ceramic foam were tested: these were 10, 20 and 30 pores per inch. The samples were cut into disks from $50 \mathrm{~mm}$ thick plates, and placed in the driven section of the shock tube as indicated in figure 5 . 


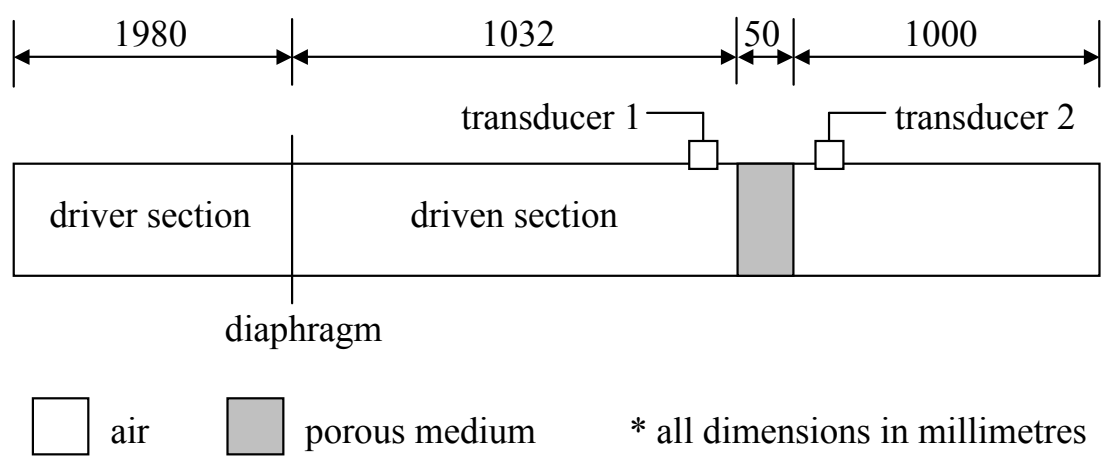

Figure 5: Schematic of experimental set-up

The three grades of porous foam were tested at three different initial shock tube diaphragm pressure ratios. The driven section of the shock tube remained at atmospheric pressure, while the driver section pressure was set to 6 bar, 5 bar and 4 bar, resulting in diaphragm pressure ratios of 6 to 1,5 to 1 and 4 to 1 . This resulted in a series of nine sets of experimental results. Each set consisted of two pressure histories, the first from transducer 1 showing the behaviour of the shock wave reflected from the front edge of the porous medium, and the second from transducer 2 showing the behaviour of waves transmitted from the back edge of the porous layer.

Each experimental set was repeated five times, although the porous sample was not changed during the repeatability tests. The reason for this was twofold: first, preliminary tests indicated that the porous material was sufficiently strong not to undergo any physical damage as a result of the interaction with the shock wave; and second, to ensure repeatability was not compromised.

Because the shock tube was constructed from a plastic material, it was susceptible to vibration. This vibration was transferred through the body of the tube to the pressure 
instrumentation. As a result of this, there is inherent noise in every experimental pressure history presented herein.

\section{Comparison between Experimental and Numerical Results}

The experimental test cases described above were simulated using the WAF based numerical formulation of the system of equations (10). In order to achieve this, it was necessary to define several key material constants relating to the three grades of porous medium. These were the Lamé constants, the Forchheimer coefficient, the tortuosity coefficient and the density of the solid substrate. The porous medium under consideration was the same material used by Levy et al (1993) in their experimental investigation of shock wave interaction with a sample of porous medium mounted at the end of a shock tube. They presented material constants for the 10, 20 and 30 ppi porous samples, which are detailed in table 2 .

Figure 6(a)-(c) illustrates a comparison between the experimental and numerical pressure histories at the first transducer position (i.e. before the porous layer). Figure 7(a)-(c) illustrates a comparison for the second transducer position (i.e. after the porous layer). A phenomenological model of the interaction of a shock wave with the front edge of a rigid porous foam is examined in detail by Levy et al (1993). This model serves to clarify the behaviour observed in the shaded regions in figure 6(a)-(c). The first shaded region shows the initial shock wave, resulting from the diaphragm burst, as it passes the first transducer. The second shows the wave reflected from the front edge of the porous layer. The third shows an increase in pressure behind the reflected wave due to reflections from internal surfaces of the porous medium, emerging from its front edge and travelling back 
up the shock tube. Figure 6(a)-(c) highlights the good correlation obtained between the experimental and numerical results.

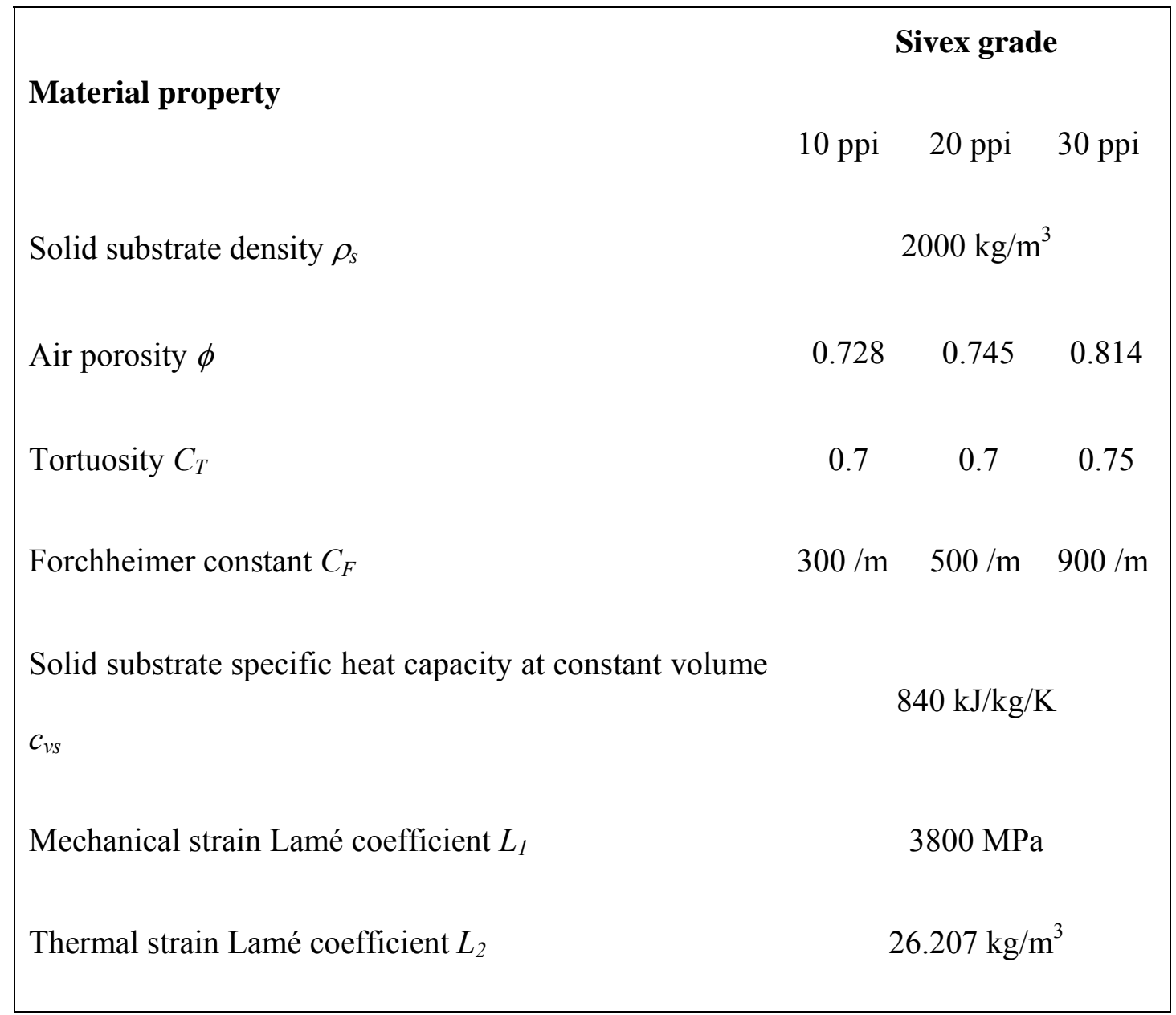

\section{Table 2: Material properties for rigid porous foam samples}

The shaded regions in figure 7(a)-(c) highlight areas of interest pertaining to the wave behaviour at the back edge of the porous sample. Figure 8(a)-(e) shows five successive time snapshots illustrating this behaviour. Figure 8(a) shows the initial compaction wave, resulting from the incidence of the initial shock wave on the porous layer, propagating towards the back edge. The second illustration shows two waves. The first is the initial compaction wave which has emerged from the porous layer and is propagating towards the second sensor. The second, represented in the figure by a broken line, is a dispersed 
compaction wave propagating towards the back edge of the porous layer. This second wave results from reflections of the primary wave from the internal surfaces of the porous medium. This concept is consistent with the phenomenological model of Levy et al (1993b).

Figure 8(c) shows a dispersed compaction wave that has just emerged from the back edge of the porous layer. Ahead of it, the initial compaction wave has reached the transducer, at which point it will perceive a pressure increase. This corresponds to the pressure jump highlighted by the first shaded area in figure 7. Figure 8(d) shows that the initial compaction wave has propagated further down the tube, while the dispersed compaction wave behind it has reached the transducer. At this point, the transducer perceives a higher pressure which corresponds to the small pressure jump highlighted in the second shaded area in figure 7. This rise is not evident in the experimental pressure histories, as the noise inherent in the signal precluded sufficient resolution of the results. However, a gradual increase in pressure from the first to the third shaded regions in figure 7(a)-(c) highlights an increase in strength of the emerging wave. 

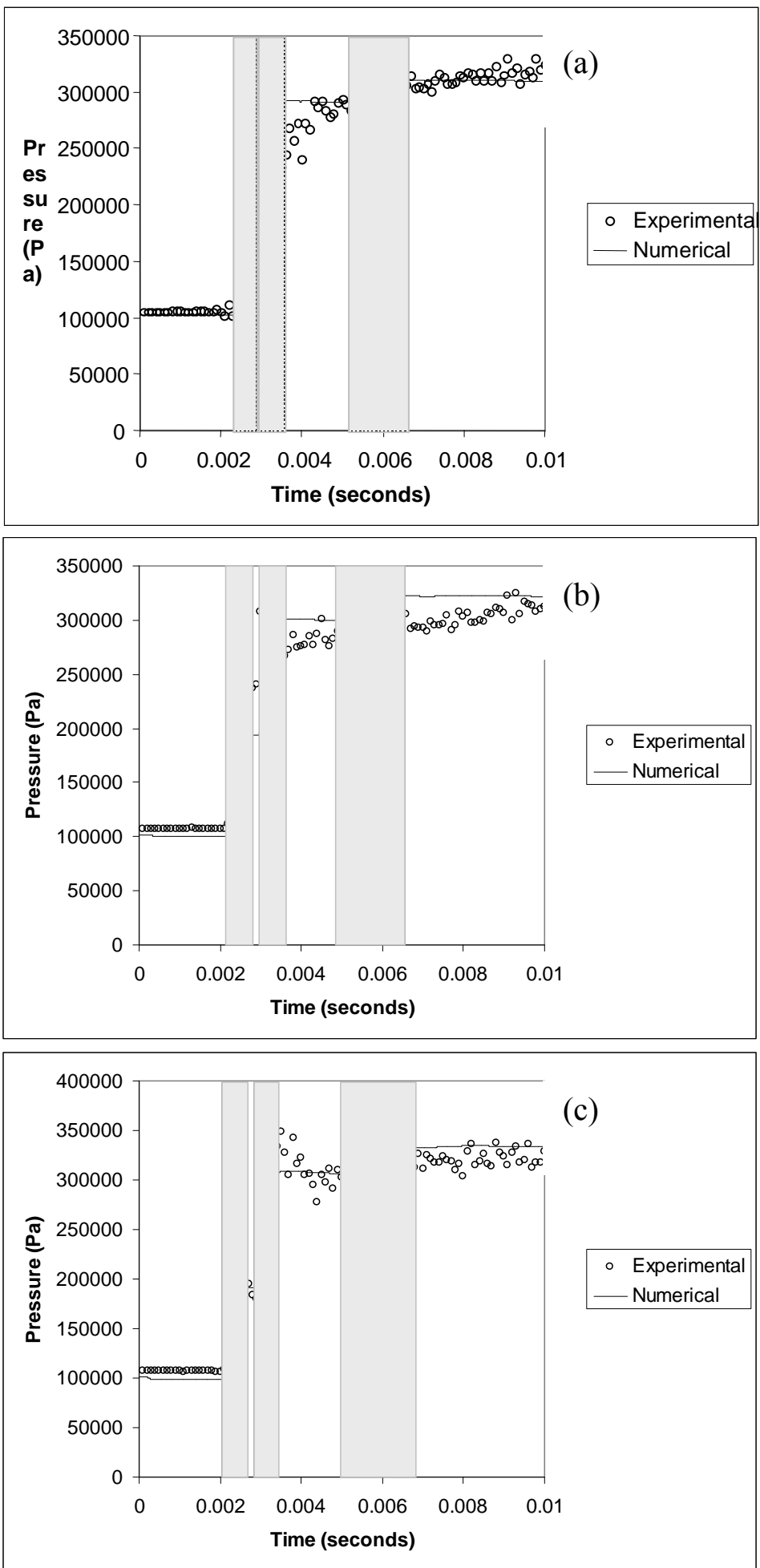

Figure 6: Pressure histories for experimental and numerical results at first transducer position: (a) 10 ppi (b) 20 ppi (c) 30 ppi 

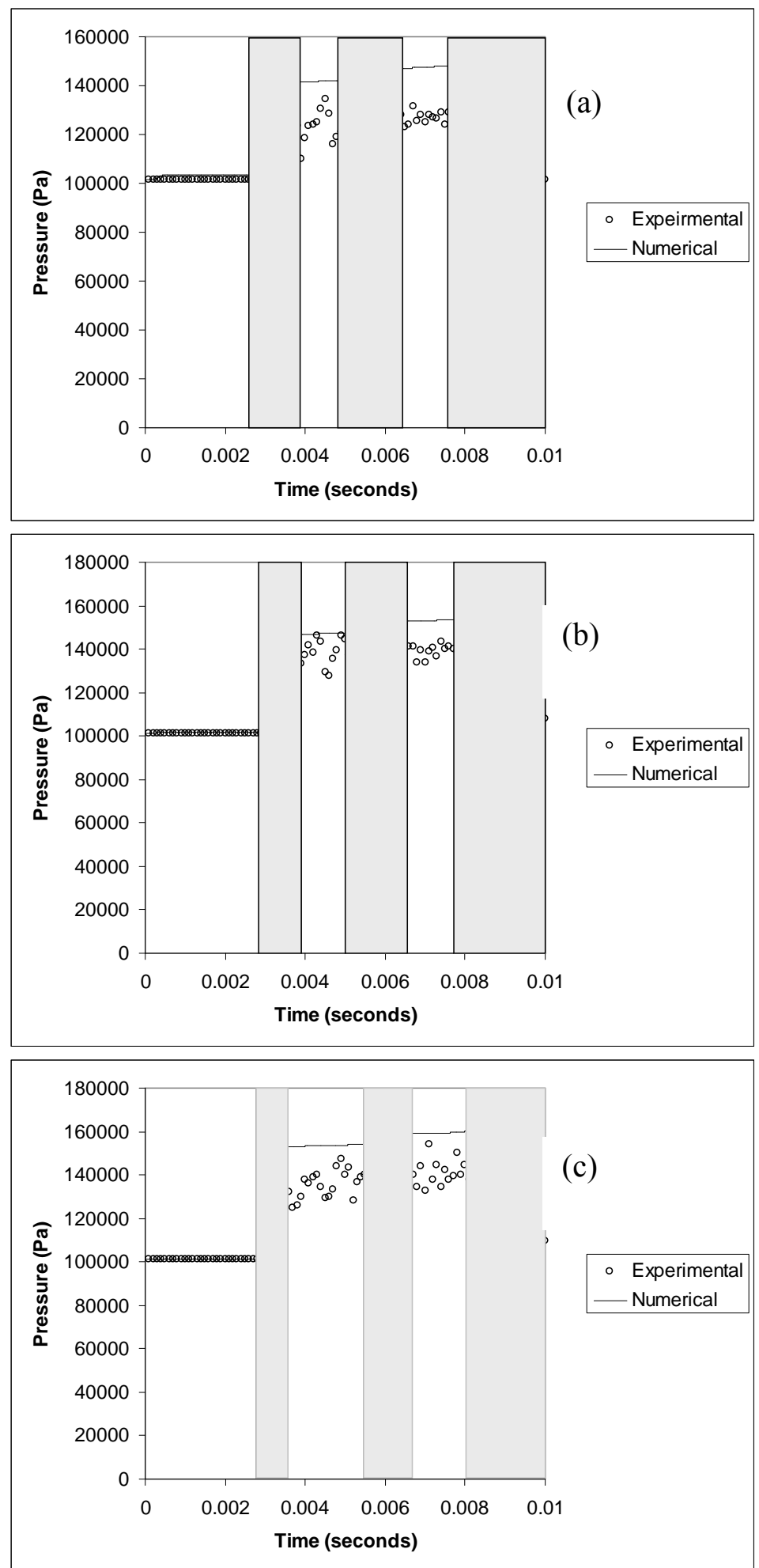

Figure 7: Pressure histories for experimental and numerical results at second transducer position : (a) 10 ppi (b) 20 ppi (c) 30 ppi 


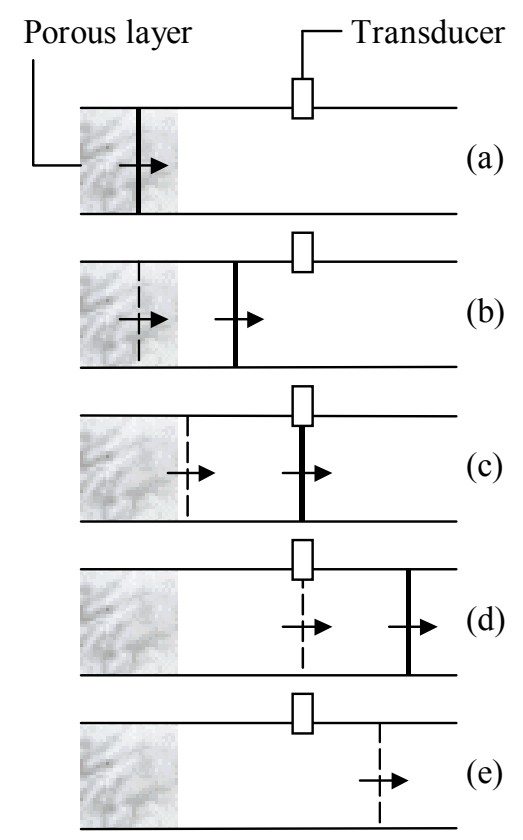

Figure 8: Wave propagation at the back edge of the porous layer

It is obvious that, when compared with the results in figure 6(a)-(c), the experimental and numerical results in figure 7(a)-(c) do not correlate as well. Although the numerical results do predict a jump in pressure, they consistently over predict the experiments by 10 to $20 \mathrm{kPa}$. The third shaded region in figure 7(a)-(c) highlights a fall off in the experimentally observed pressure which is not mirrored by the numerical results.

The primary reason the numerical results over predict the experimental results lies in some of the assumptions made to simplify the system of governing equations in order to produce the numerical model (Torrens and Wrobel (2002)). Of the assumptions made, the two of most interest are (a) the fluid is ideal and therefore does not experience any viscous dissipative forces and (b) the energy processes in the fluid and the solid are reversible. 
Consider firstly assumption (a). On entering the porous layer, the shock wave is subject to many internal surfaces with which it may interact. Levy et al (1995) present an evolution on the governing macroscopic balance equations. They identified four distinct time periods in which the behaviour of the equations changes from convection-dominated to diffusion-dominated. The first is a period of uniform pressure, temperature and stress distribution. This occurs at the instant the porous medium is subjected to the abrupt change in pressure. The second is a period of non-linear wave propagation that is dominated by convection. This is characterised by a sharp, well-defined compaction wave propagating in the porous medium. This is the time period that is most effectively modelled by the numerical model presented here. The third is a period in which dissipative effects start to become apparent. The compaction wave in the porous layer starts to be more affected by the interaction with the internal surfaces of the porous medium. The final period is dominated by dissipative terms. The viscous dissipation caused by friction on the internal surfaces characterises the flow. The behaviour of the flow during the third and fourth evolutionary periods contradicts the Eulerian flow assumption made by the mathematical model.

Adherence to assumption (b) would mean that the flow is assumed to be isentropic (i.e. no energy is lost to the environment due to friction). However, from the discussion presented above, it is clear that if the flow is in either the third of forth evolutionary period, then viscous dissipation (or drag) on the internal surfaces of the porous medium would play a role in characterising the flow. This viscous drag would render the flow anisentropic. Neglecting the viscous dissipation or the anisentropic nature of the flow 
could account for a proportion of the over prediction of the numerical results that was observed in figure 7(a)-(c).

The second discrepancy between the numerical and experimental results presented in figure 7(a)-(c) relates to the fall in pressure observed in the third shaded region. This phenomenon occurred consistently throughout all of the transducer two pressure histories for all test cases. The cause of this would be closely linked with the explanation for the over prediction of numerical results given above.

\section{Phenomenological Model}

Skews et al (1992) presented a phenomenological model to account for the behaviour of compressible porous foams under shock loading conditions. This model was extended by Levy et al (1993) to explain experimental observations made for shock wave interaction with rigid porous foams, for the specific case of the porous medium shock tube problem configuration illustrated in figure 2(c) where a porous medium sample is mounted in a shock tube with its back edge flush with the tube end wall. Figure 9 shows a characteristic plot illustrating this configuration.

Four types of characteristics can be seen in figure 9. These are the initial shock wave incident on the front edge of the porous medium $\left(S_{I}\right)$, the reflected shock wave $\left(S_{R}\right)$, the compaction wave transmitted into the porous medium $\left(C_{T}\right)$, and the dispersed compaction waves resulting from reflections from internal surfaces $\left(C_{R}\right)$. The latter type of characteristic is shown by broken lines. Part of these waves propagate towards the end wall and are reflected, while compaction waves are reflected out of the front edge of the porous medium. It can be seen in the figure that the reflected shock wave is strengthened 
as a result of the superposition of the compaction waves emerging from the front edge of the porous sample.

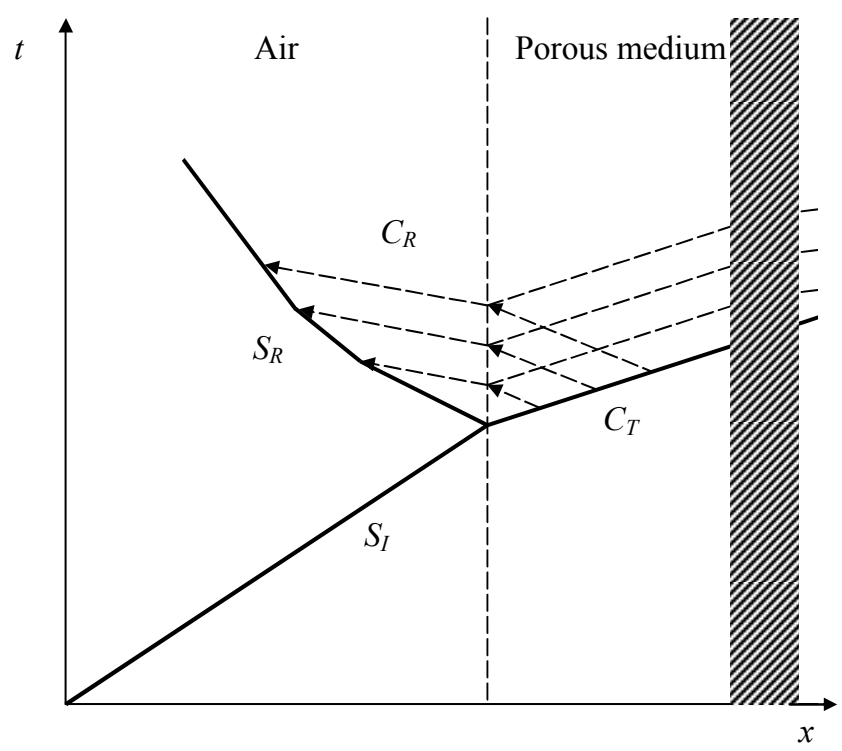

Figure 9: Characteristic $x$-t diagram for phenomenological model of Levy et al. (1993)

Figure 10 shows a characteristic diagram of the proposed extension to the phenomenological model of Levy et al (1993). In this case, five distinct waves groups appear in figure 10, the same four as in figure 9 and an extra wave transmitted from the back edge of the porous layer $\left(S_{T}\right)$. The dispersed compaction waves are shown as broken lines.

The extension of the model lies in the transmission of the compaction wave $\left(C_{T}\right)$ from the back edge of the porous sample back into the single-phase shock tube. The main compaction wave transmitted through the porous medium emerges from the back edge and forms a shock wave $\left(S_{T}\right)$ which continues to propagate down the shock tube. The forward running dispersed compaction waves emerge from the back edge of the porous 
medium. As with the reflected shock wave, these dispersed compaction waves serve to strengthen the transmitted shock wave. This extension to the phenomenological model is based on observations made of the sensor two pressure histories previously shown.

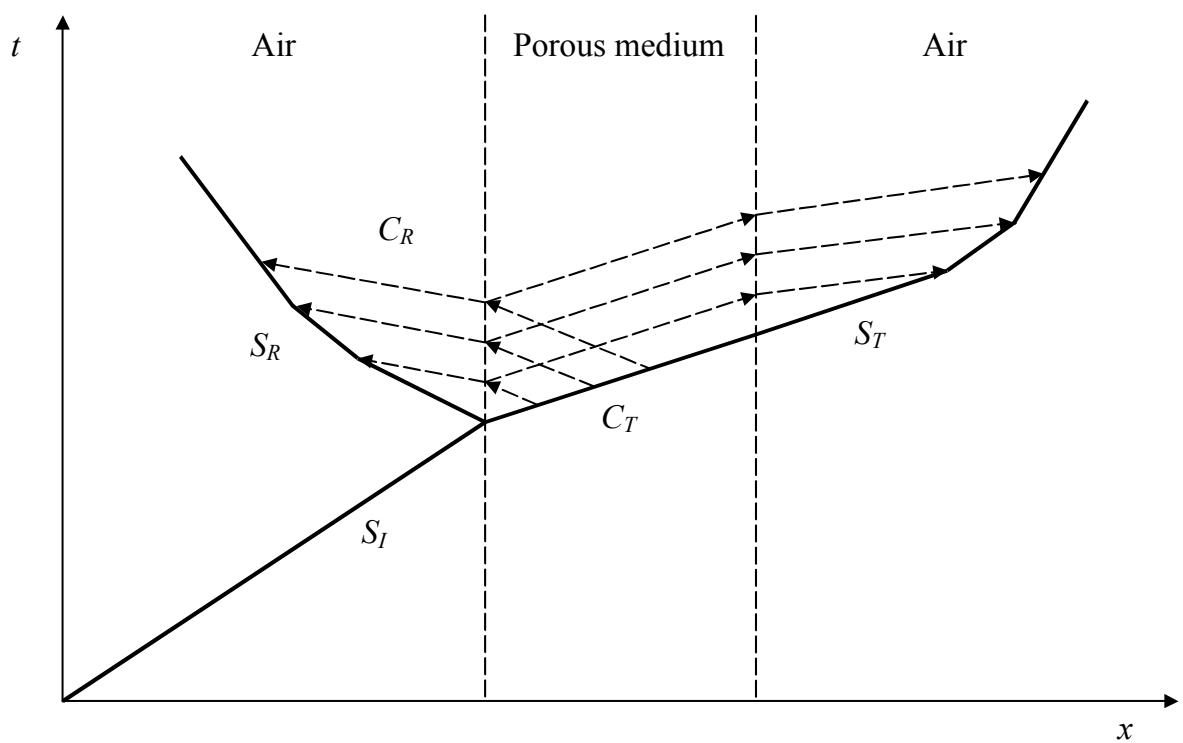

Figure 10: Characteristic $x-t$ diagram for proposed phenomenological model

\section{Conclusions}

A TVD flux limited WAF-based numerical formulation of the two phase macroscopic balance equations has been utilised to carry out a study of shock wave propagation through a layer of a rigid porous medium. This differed from previous studies in that the compaction wave propagating through the porous layer was transmitted into the single phase flow at the back face of the porous layer. A shock tube based experimental study was performed for the purpose of validating the numerical model. 
Excellent agreement was observed between the experimental and the numerical results for the wave reflected from the front edge of the porous layer. However, the correlation between the experimental and numerical results of the wave transmitted from the back edge of the porous layer was not as good. This was thought to be caused by modelling assumptions that result in neglecting viscous dissipation and flow irreversibilities that occur within the porous medium.

The increase in strength of the wave transmitted from the back edge of the porous layer was caused by the accumulation of dispersed compaction waves resulting from reflections from the internal surfaces of the porous layer. This inference led to the phenomenological model that has been proposed to describe the wave behaviour before and after the porous sample.

\section{References}

Baer M. R. (1988). Numerical studies of dynamic compaction of inert and energetic granular materials. Transactions of the ASME, Journal of Applied Mechanics 55(1): 3643.

Bear J. and Bachmat Y. (1990). Introduction to Modelling Transport Phenomena in Porous Media. Kluwer Academic Publishers.

Bear J. and Sorek S. (1990). Evolution of governing mass and momentum balances following an abrupt pressure impact in a porous-medium. Transport in Porous Media 5(2): 169-185. 
Biot M. A. (1956). Theory of propagation of elastic waves in a fluid-saturated porous solid. I Low-frequency range. Journal of the Acoustical Society of America 28(2): 168178.

Corapicoglu M. Y. (1991). Wave propagation in porous media - a review. Transport Processes in Porous Media (Editors J. Bear and M. Y. Corapicoglu). Kluwer Academic Publishers.

Harten A. (1983). High-resolution schemes for hyperbolic conservation-laws. Journal of Computational Physics 49(3): 357-393.

Hassanizadeh S. M. and Gray W. G. (1979a). General conservation equations for multiphase systems. I. Averaging procedure. Advances in Water Resources 2(3): 131144.

Hassanizadeh S. M. and Gray W. G. (1979b). General conservation equations for multiphase systems. II. Mass, momentum, energy and entropy equations. Advances in Water Resources 2(4): 101-203.

Hassanizadeh S. M. and Gray W. G. (1980). General conservation equations for multiphase systems. III. Constitutive theory for porous media flow. Advances in Water Resources 3(1): 25-40.

Kistler Instruments Ltd (2000), Private communication.

Levy A., Ben-Dor G., Skews B. and Sorek S. (1993). Head-on collision of normal shockwaves with rigid porous materials. Experiments in Fluids 15(3): 183-190.

Levy A., Ben-Dor G. and Sorek S. (1996). Numerical investigation of the propagation of shock waves in rigid porous materials: Development of the computer code and comparison with experimental results. Journal of Fluid Mechanics 324: 163-179. 
Levy A., Sorek S., Ben-Dor G and Bear J. (1995). Evolution of the balance equations in saturated thermoelastic porous media following abrupt simultaneous changes in pressure and temperature. Transport in Porous Media 21(3): 241-268.

Powers J. M., Stewart D. S. and Krier H. (1989). Analysis of steady compaction waves in porous materials. Transactions of ASME, Journal of Applied Mechanics 56(1): 15-24.

Roe P. L. (1981). Approximate Riemann solvers, parameter vectors, and differenceschemes. Journal of Computational Physics 43(2): 357-372.

Roe P. L. (1983). Some contributions to the modelling of discontinuous flows. SIAM/AMS Seminar, San Diego.

Rogg B., Hermann D. and Adomeit G. (1981). Shock induced flow in a porous medium. Proceedings of EuroMech, Delft.

Skews, B. W., M. D. Atkins and Seitz M. W. (1992). Gas dynamic and physical behaviour of compressible porous foams struck by a weak shock wave. Shock Waves. 18th International Symposium, Sendai, Japan.

Toro E. F. (1992). The weighted average flux method applied to the Euler equations. Philosophical Transactions of the Royal Society of London, Series A 341(1662): 499-530. Toro E. F. (1999). Riemann Solvers and Numerical Methods for Fluid Dynamics. Springer Verlag.

Torrens R. and Wrobel L.C. (2002). Weighted average flux method and flux limiters for the numerical simulation of shock waves in rigid porous media. International Journal for Numerical Methods in Fluids 40: 1187-1207. 